\section{Dental implants and squamous cell carcinoma in the at risk patient - report of three cases}

\author{
J. Kwok, ${ }^{1}$ J. Eyeson, ${ }^{2}$ I. Thompson ${ }^{3}$ and M. McGurk ${ }^{4}$
}

IN BRIEF

- Clinicians need to be aware that squamous cell carcinoma (SCC) can complicate dental implant patients.

- Prompt instigation of oral hygiene measures for inflamed peri-implant tissue is especially important in individuals with risk factors for SCC.

- There should be a low threshold for biopsy of persistent inflamed peri-implant tissue not responding to local oral hygiene measures in individuals at risk of SCC.

Osseointegrated dental implants are increasingly used in the rehabilitation of the dental patient. They have a particular role in dental rehabilitation following treatment for oral cancer. Data is presented that suggests that, in the at risk patient, squamous cell carcinoma may develop in association with dental implants.

\section{INTRODUCTION}

Dental implants have revolutionised the practise of dentistry and are now an integral part of modern clinical practice. In addition, implant-based restorations (dentures, obturators or crowns) have a beneficial role in the dental rehabilitation of patients treated surgically for oral cancer. ${ }^{1,2}$

In such circumstances, the tissues are frequently distorted, scarred and insensate, which makes it almost impossible to retain a traditional lower denture. Quality of life measures show that patient well-being is significantly improved by restoring dental function.

The pathogenesis of oral squamous cell carcinoma (SCC) is well established. In humans it is linked largely to lifestyle habits, in particular the excess consumption of alcohol and tobacco. Pre-malignant lesions are well characterised in the form of some leukoplakias and erythroplakia. Occasionally patients with lichen planus can develop the disease. ${ }^{3}$ The disease can also evolve de novo in the elderly, without apparent risk factors apart from the biological effects of age.

\footnotetext{
Associate Specialist, ${ }^{2}$ Lecturer, ${ }^{3}$ Research Fellow

${ }^{4}$ Professor, Department of Oral \& Maxillofacial Surgery, King's College London Dental Institute, Floor 23, Guy's Tower, London, SE1 9RT

${ }^{*}$ Correspondence to: Dr Josiah Eyeson

Email: josiah.eyeson@kcl.ac.uk
}

\section{Refereed Paper}

Accepted 20 August 2008

DOI: $10.1038 /$ sj.bdj.2008.980

${ }^{\circledR}$ British Dental Journal 2008; 205: 543-545
In the animal model, squamous cell carcinoma can be reliably induced by a two stage process. The first involves priming the oral mucosa with a carcinogenic agent. After a period of delay this primed mucosa can be induced to develop SCC by a seemingly innocent 'initiating' factor such as trauma or irritation. In this context, dental implants lack an epithelial attachment at the cervical margin and this is a recognised source of persistent irritation which normally has no serious implications apart from for those predisposing to peri-implantitis.

This paper reports three patients with implant-based restorations who subsequently developed SCC in the periimplant tissue. Two cancers arose de novo. The implications are discussed.

\section{CASE 1}

A 62-year-old male patient with poorly fitting upper and lower dentures sought advice regarding implant retained prostheses. His medical history was unremarkable except for a history of excessive alcohol and tobacco consumption. The patient admitted to smoking 40 cigarettes a day for over 30 years but stopped two months prior to his attendance. After appropriate assessment and surgical planning, the upper and lower arches were restored with fixed prostheses supported on dental implants. Surgery was undertaken via a flapless procedure with eight Branemark implants placed in the upper arch and six in the lower arch.
The final restoration was completed without complications.

Subsequently at a routine review appointment three months after completion of treatment, a non-healing ulcer was evident in the peri-implant tissue on the lingual aspect of the mandible (lower right premolar region). The ulcer was biopsied and reported as moderately well differentiated SCC.

The patient was subsequently referred to the Head and Neck Oncology Centre, Guy's Hospital, London, where he underwent treatment for the tumour. The patient made an uneventful recovery from his surgery but three years later unfortunately succumbed to a second cancer arising in the stomach.

\section{CASE 2}

A 71-year-old professional man had a history of poor retention of his lower denture. He requested an implant retained prosthesis for both comfort and masticatory function. His medical history was unremarkable except that he had previously smoked 40 cigarettes a day and had stopped the habit 15 years previously. However, he drank to excess (20 units of alcohol per day). The treatment plan consisted of an implant supported lower denture with two Branemark implants.

The patient attended regular review appointments. After a period of six years he developed what appeared to be inflammatory changes around one of the dental implants. This failed to settle with local measures and a biopsy 
demonstrated a well differentiated SCC. The patient was treated surgically but a perforated duodenal ulcer in the immediate postoperative period proved fatal.

\section{CASE 3}

A 67-year-old female had a history of two small squamous cell carcinomas on the lateral border of her tongue. These were treated by local excision and direct repair in 2001 and 2004. She had also been treated successfully for breast carcinoma in 1992. She had previously smoked approximately 40 cigarettes a day for 30 years but had not smoked since 1997. She consumed up to seven units of alcohol per week.

The patient was edentulous and as a result of the previous surgical treatment she was unable to control her lower denture. An implant borne lower denture using two implants was provided in 2005 and proved a great success.

In 2006 the patient developed an area of papillary hyperplasia on her lower lip which was an outward and visible sign of the general biological instability of her oral mucosa. A lip shave was performed but at surgery an incidental finding was a small area of granulation tissue around the lower left implant. A biopsy revealed early SCC. The tumour was clearly associated with the implant and could not be considered a recurrent tumour. Treatment was by local excision and the patient remains disease-free.

\section{DISCUSSION}

Osseointegrated dental implants have revolutionised modern dentistry, in particular the restoration of edentulous or partially edentulous jaws. Implants also have an important place in the restoration of dental function and aesthetics after the surgical treatment of oral tumours. It is well established that patients with oral SCC are at increased risk ${ }^{4}$ of developing a new second primary tumour and as in case 3 , they may occur in patients restored with implants. The presence of an implant may have been an incidental factor. But two patients developed SCC de novo directly within the cuff of periimplant tissue, raising the possibility of a relationship between peri-implantitis and tumour.
The nature of gingival attachment is a recurring problem with dental implants, which is reflected in the range of different implant designs. This area of persistent low grade inflammation normally has few or no serious implications on the biological stability of the oral mucosa.

Both alcohol and tobacco use are known risk factors for SCC. ${ }^{5}$ These also compromise the healing process ${ }^{6}$ and increase the risk of implant failure. ${ }^{7-11}$ It has been suggested that smokers are informed of this risk rather than smoking being seen as a contraindication to implant treatment. ${ }^{12}$

All three patients had a history of cigarette smoking and two had high alcohol consumption, and they represented high risk cases for developing oral cancer. The point of interest is that all tumours occurred in the peri-implant tissue cuff. The implication is that occasionally in the at risk patient, cancerous change may occur in peri-implant tissues and mimic peri-implantitis. Consequently it is important to be familiar with patient risk profiles and have a low threshold for biopsy in this patient group.

Ten cases of SCC developing around peri-implant tissues have been reported in the literature. Only three cases reported previous oral SCC resection. Clapp et $a l{ }^{13}$ reported on three cases, one of whom had no history of known risk factors. The second case had moderate dysplasia of the oral mucosa and the third case a history of previous oral SCC resection. Block et al. ${ }^{14}$ reported one case that developed oral SCC five months after implant provision. He had given up smoking 16 years earlier but had recurrent verrucous carcinoma, a pre-malignant lesion, over the preceding 11 years. Shaw et al. ${ }^{15}$ reported two cases of oral SCC in people who had previously undergone oral SCC resection. Recently, two cases (one was a heavy smoker) of squamous cell carcinoma mimicking peri-implantitis have been reported. ${ }^{16}$ All but two of the 13 patients, including the three in our series, had reported risk factors.

Inflammation has been shown to play an important role in carcinogenesis via mediators such as oxidants, prostaglandins, interleukin-1, interleukin-6 and tumour necrosis factor. ${ }^{17,18}$ Inflammatory changes around implants (peri-implantitis) are well known phenomena ${ }^{19}$ and in theory could act as an initiating agent for malignancy in an already 'primed mucosa'. Dental implants in themselves have no known malignant potential and have been categorised in Group 3 (not classifiable as to their carcinogenicity to humans) in an evaluation of the carcinogenic risks to humans associated with surgical implants. ${ }^{20}$

Although it is difficult to know whether the present individuals would have developed a malignancy in the absence of dental implants, the present cases raise the possibility that in at risk groups, irritation around implants may act as a factor associated with carcinogenesis, a point highlighted by Czerninski et al. ${ }^{21}$ With the increased provision of dental implants within the population, the frequency of this occurring is likely to increase. It is not the intention of this paper to advise against the use of implants in this at risk population but only to highlight the possibility of such a situation occurring and to advise a low threshold for biopsy in this at risk population when inflamed peri-implant tissues persist following adequate local oral hygiene measures.

1. Weischer T, Mohr C. Ten-year experience in oral implant rehabilitation of cancer patients: treatment concept and proposed criteria for success. Int J Oral Maxillofac Implants 1999; 14: 521-528.

2. Shaw R J, Sutton A F, Cawood J I et al. Oral rehabilitation after treatment for head and neck malignancy. Head Neck 2005; 27: 459-470.

3. Van der Meij E H, Mast H, van der Waal I. The possible premalignant character of oral lichen planus and oral lichenoid lesions: a prospective five-year follow-up study of 192 patients. Oral Oncol 2007; 43: 742-748.

4. Jovanovic A, van der Tol I G, Kostense P J et al. Second respiratory and upper digestive tract cancer following oral squamous cell carcinoma: oral oncology. Eur J Cancer 1994; 30B: 225-229.

5. Johnson N W, Ranasinghe A W, Warnakulasuriya K A A S. Potentially malignant lesions and conditions of the mouth and oropharynx: natural history - cellular and molecular markers of risk. Eur J Cancer Prev 1993; 2(Suppl 2): 31-51.

6. Reibel J. Tobacco and oral diseases: update on the evidence with recommendations. Med Princ Pract 2003; 12: 22-32.

7. Bain C A, Moy P K. The association between the failure of dental implants and cigarette smoking. Int J Oral Maxillofac Implants 1993; 8: 609-615.

8. De Bruyn H, Collaret B. The effects of smoking on early implant failure. Clin Oral Implants Res 1994; 5: $260-264$.

9. Lambert P M, Morris H F, Ochi S. The influence of smoking on 3-year clinical success of osseointegrated dental implants. Ann Periodontol 2000; 5: 79-89.

10. Schartz-Arad D, Samet N, Samet N, Mamlider A. Smoking and complications of endosseous dental implants. J Periodonto/ 2002; 73: 153-157. 
11. Galindo-Moreno P, Fauri M, Avila-Ortiz G, Fernandez-Barbero J E, Cabrera-Leon A, Sanchez-Fernandez $\mathrm{E}$. Influence of alcohol and tobacco habits on peri-implant marginal bone loss: a prospective study. Clin Oral Implants Res 2005; 16: 579-586.

12. DeLuca S, Zarb G. The effect of smoking on osseointegrated dental implants. Part II: periimplant bone loss. Int J Prosthodont 2006; 19: 560-566

13. Clapp C, Wheeler J C, Martof A B, Levine P A. Oral squamous cell carcinoma in association with dental osseointegrated implants. Arch Otolaryngol Head Neck Surg 1996; 122: 1402-1403.

14. Block M S, Scheufler E. Squamous cell carcinoma appearing as peri-implant bone loss; a case report.
J Oral Maxillofac Surg 2001; 59: 1349-1352.

15. Shaw R, Sutton D, Brown J, Cawood J. Further malignancy in field change adjacent to osseointegrated implants Int J Oral Maxillofac Surg 2004; 33: 353-355.

16. Abu El-Naaj I, Trost O, Tagger-Green N et al. Peri-implantitis or squamous cell carcinoma? Rev Stomatol Chir Maxillofac 2007; 108: 458-460.

17. Weitzman S A, Gordon L I. Inflammation and cancer: role of phagocyte-generated oxidants in carcinogenesis. Blood 1990; 76: 655-663.

18. Jiiang-Huei J, Ying-Jan W, Bor-Luen C et al. Roles of keratinocyte inflammation in oral cancer: regulating the prostaglandin $\mathrm{E} 2$, interleukin- 6 and TNF-a production of oral epithelial cells by areca nut extract and arecoline. Carcinogenesis 2003; 24: 1301-1315.

19. Esposito M, Worthington H V Coulthard P. Interventions for replacing missing teeth; treatment of peri-implantitis. Cochrane Database Syst Rev 2004; 4: CD004970.

20. McGregor D B, Baan R A, Partensky C, Rice J M, Wilbourn J D. Evaluation of the carcinogenic risks to humans associated with surgical implants and other foreign bodies - a report of an IARC Monographs Programme Meeting. Eur J Cancer 2000: 36: 307-313.

21. Czerninski R, Kaplan I, Almoznino G, Maly A, Regev E. Oral squamous cell carcinoma around dental implants. Quintessence Int 2006; 57: 707-711. 